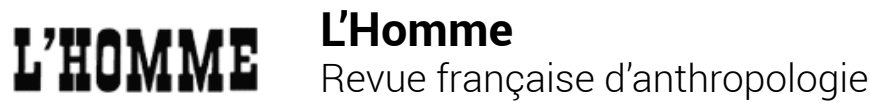

163 | juillet-sptembre 2002

De la légende au mythe. Parole, langue et pensée

\section{La transcendance de la courgette, ou les dieux nécessaires}

\section{Marie-Claude Dupré}

\section{(2) OpenEdition \\ Journals}

\section{Édition électronique}

URL : http://journals.openedition.org//homme/12341

DOI : 10.4000//homme.12341

ISSN : 1953-8103

Éditeur

Éditions de l'EHESS

\section{Édition imprimée}

Date de publication : 21 juin 2002

Pagination : 235-244

ISBN : 2-7132-1771-7

ISSN : 0439-4216

\section{Référence électronique}

Marie-Claude Dupré, "La transcendance de la courgette, ou les dieux nécessaires », L'Homme [En ligne], 163 | juillet-sptembre 2002, mis en ligne le 03 juillet 2007, consulté le 24 septembre 2020. URL: http://journals.openedition.org//homme/12341 ; DOl : https://doi.org/10.4000//homme.12341 


\title{
La transcendance de la courgette, ou les dieux nécessaires
}

\author{
Marie-Claude Dupré
}

\begin{abstract}
$\mathrm{N}$ AGUÈRE, «l'esprit humain était semblable à ces vastes territoires inexplorés de l'Afrique remplis de crocodiles et de palmiers sur les cartes anciennes" (p. 11). Pascal Boyer propose dans son livre une description dynamique du fonctionnement de l'esprit humain, alliant «l'évolution, la biologie, la psychologie, l'archéologie et l'anthropologie» (p. 294), ce qui nous vaut une conjonction inédite de travaux récents dans ces domaines. Le projet est novateur, stimulant, apte à sortir l'expérience anthropologique de ses sentiers battus. L'auteur montre comment la religion, universellement présente dans les sociétés humaines, plonge ses racines dans l'exercice même de l'activité de l'esprit. Ce terme est toutefois quelque peu trompeur dans la traduction française d'un livre dont nous ne connaissons pas le titre en anglais; il s'agit très probablement ici du "brain ", du cerveau matériel et corporel.

Pascal Boyer entend explorer le "sous-sol » de nos réactions cognitives dont notre cerveau est le siège, réactions qui échappent à la conscience parce que trop rapides et simultanées. Notre cerveau, en effet, est riche de réflexes chimiques, électriques, hormonaux dont l'agencement, au moment de la naissance, est plus opératoire qu'on ne l'a cru jusqu'à présent. Un cerveau, donc, préfiguré, préparé à faire jouer les divers «systèmes d'inférence " qui président au développement des processus cognitifs conscients. Cette exploration s'appuie sur des expériences de psychologie sociale menées exclusivement, si l'on se réfere à la bibliographie, dans les universités anglophones. Il faut préciser ici que ces expériences saisissent l'activité mentale à travers des résolutions de problèmes ou des constructions de consensus, ou encore des "tâches simples" (p. 283) assignées à des individus arbitrairement répartis en deux groupes. Le point important, qui échappe en grande partie à la présentation des résultats et à l'utilisation « théorique " généra-
\end{abstract}


lisante qui en est faite, est qu'il s'agit toujours d'actions de coopération positive entre inconnus rassemblés en territoire neutre en vue d'un but principal, sinon unique. Visant à mettre en lumière les réflexes activés par une interaction individuelle, ces actions sont censées rendre observables les dynamismes sociaux dans leur état originel en les dégageant de toute pesanteur sociologique, et (donc ?) en les faisant se manifester à un stade pré-social, pré-conscient et a-temporel. Le lecteur ethnologue note que la division arbitraire en deux groupes étiquetés bleus et rouges, à l'instar des équipes de foot (ou plutôt de baseball), active en fait des réflexes sociaux et sportifs propres aux Américains actuels. Qu'importe! Les observations obtenues sont supposées restituer l'émergence de la sociabilité. Pour l'anthropologue, plus accoutumé à observer les pesanteurs de l'histoire et des hiérarchies sociales, ce cadre expérimental, non explicité par Pascal Boyer, est à priori déroutant. Il ne correspond pas à ses observations de terrain où les contraintes sociologiques façonnent les individualités et assurent la permanence de sociétés originales.

La force démonstrative de l'étude est plus stimulante lorsqu'elle surgit de la déconstruction d'ensembles culturels que l'on croyait bien connaître dans leur diversité, comme la variabilité des formes religieuses. Pascal Boyer commence son périple en réduisant la richesse ethnologique, adossée à plusieurs décennies de recherches, à quelques caractéristiques générales à la fois provocatrices et lumineuses qu'il énumère dans le premier chapitre, intitulé "La question des origines ". Les douze scénarios qui résument toutes les spéculations sur l'origine de la religion sont répartis en quatre sous-ensembles - l'explication, le réconfort, l'ordre social et l'illusion (p. 13) - dont il montre qu'aucun n'est satisfaisant. Il décide alors de "prendre le problème à l'envers» (p. 36), c'est-à-dire de partir non pas des besoins sociaux ou métaphysiques, mais des systèmes d'inférence cognitifs présents dans "l'architecture mentale " du cerveau, systèmes dont le travail échappe à l'appréhension consciente et dont le fonctionnement est révélé par les processus expérimentaux de la psychologie sociale. Mais, malgré l'avalanche des résultats d'expérience et d'une copieuse bibliographie, le lecteur, même non anthropologue, en vient à se demander pourquoi l'activité cognitive supposée du chasseur-cueilleur préhistorique sert de modèle aux protocoles de laboratoire. Il s'émerveille d'apprendre que les milliards de messages émis et perçus sans conscience, les confrontations effectuées à la vitesse de la lumière entre des systèmes d'inférences qui se hiérarchisent pour répondre à une stimulation précise, servent uniquement (ou principalement) à deviner comment l'autre va contribuer à une action nécessairement conjointe, et, s'agissant du mâle préhistorique, assurer le succès de l'entreprise cynégétique (et pourquoi la femme cueilleuse ne sert-elle pas aussi de modèle ?).

Lévolutionnisme, autre pilier de l'anthropologie cognitive, semble négliger le temps, relativement court il est vrai, qui nous sépare de l'homme préhistorique, lequel doit faire preuve en permanence de sa "fiabilité » (p. 183) et utiliser l'excitation de ses systèmes d'inférence pour deviner la construction cognitive de l'opinion des autres et agir en conséquence. Ce serait, par ailleurs, toujours nos réflexes cognitifs de chasseur-cueilleur, prédateur menacé risquant à chaque instant de se 
transformer en proie, qui s'exercent à l'insu de notre conscience et qui sont présentés à plusieurs reprises comme le substrat des sociétés modernes (p. 145). Aujourd'hui comme hier, et c'est encore l'ethnologue qui déduit cette proposition d'un ensemble de résultats "expérimentaux ", l'être humain doit sa survie à sa capacité d'engager des interactions qui le définissent comme une "espèce de coopérateurs» (p. 189). Le lecteur ethnologue note toutefois avec un peu de surprise une différence importante entre les techniques de chasse observées chez les chasseurs-cueilleurs contemporains et le modèle de coopération qui se trouve au fondement des spéculations cognitives et des expériences de laboratoire. Pour la psychologie sociale, tout se passe comme si les techniques de chasse préhistoriques rassemblaient des partenaires qui ne se connaissaient pas et passaient leur temps à imaginer les pensées des uns et des autres. L'auteur ne se réfere pas à la documentation existante qui décrit la chasse comme une opération complexe, très strictement réglementée, afin justement de diminuer au maximum l'effet négatif produit par une compétition élémentaire qui réserverait la capture du gibier toujours au même acteur. Des précautions à la fois rituelles et techniques assurent l'égalité entre chasseurs, par rotation des postes par exemple, et visent à neutraliser les "pouvoirs" magiques qui risquent de déséquilibrer l'accès à la ressource. Autrement dit, quelques lectures judicieuses montreraient que la situation de compétition pure et neutre postulée chez les chasseurs préhistoriques (car la situation est envisagée au niveau zéro de la construction sociale) est en réalité le résultat recherché de techniques sociales bien contrôlées, et aucunement le fait d'une sociabilité à peine émergente ${ }^{1}$. À nouveau, une activité humaine réduite à sa facette positive, utile, est dépouillée de sa dimension sociale (il n'existe pas d'humain isolé) en niant qu'un travail réflexif puisse être effectué par les acteurs sur leur activité. Pourtant, tenir compte de la subtilité psychologique incluse dans l'organisation d'une chasse collective n'infirmerait pas l'investigation cognitive; elle montrerait seulement que les humains n'ont pas attendu les expérimentateurs américains pour activer leurs réflexes cognitifs de façon positive, en inhibant ceux qui pourraient nuire à la "tâche simple " d'une chasse au filet.

Ignorer (volontairement ?) que la psychologie «naïve» d'Homo cynegeticus est opérationnelle parce qu'elle suscite une organisation qui privilégie l'effet positif de la coordination des tâches risque de faire disparaître du champ d'étude d'autres manifestations socialement négatives, telle la sorcellerie, que les lois de l'évolution ne prennent pas en compte mais qui, curieusement, se maintiennent jusqu'à présent et connaissent même un regain d'activité. C'est pourtant ainsi que commence le livre, avec l'évocation des «flèches invisibles » chez les Fang (du Cameroun) (p. 9). En fait, la sorcellerie ne peut être mise en scène par les psychologues américains, car bien qu'elle "se retrouve dans à peu près tous les groupes humains, sous différentes formes » (p. 191), comme en convient Pascal Boyer, elle n'est qu'un « soupçon » que l'enquête anthropologique ne peut confirmer, puisqu'il s'agit d'une activité anti-

1. Georges Dupré, Un ordre et sa destruction. Économie, politique et histoire chez les Nzabi de la République populaire du Congo, Paris, Éditions de l'ORSTOM, 1982. On peut dire que les Nzabi ont les mêmes techniques de base que les Fang chez lesquels Pascal Boyer a commencé sa carrière d'anthropologue. 
sociale par excellence que personne ne veut assumer (ibid.). L'auteur renvoie ici au livre de Jeanne Favret-Saada ${ }^{2}$ qui a travaillé dans le "Bocage ", pays non identifié dans le livre (ibid.). S'agissant d'un ouvrage en français, le Bocage pourrait être situé dans un de nos anciens comptoirs de l'Inde, en Suisse ou au Canada... Et comme, de toutes façons, selon les témoignages des anthropologues vaguement évoqués par Pascal Boyer, « les menées anti-sorciers sont des opérations de sorcellerie » (p. 190), l'auteur dispose d'un moyen élégant pour inclure ces actions socialement négatives dans l'ensemble coopération qui reste ainsi globalement positif. La sorcellerie étant par ailleurs une théorie du malheur, tout comme la religion qui justifie l'existence d'ancêtres mécontents et de dieux vengeurs, elle se voit écartée du champ de l'étude par cette définition spéculative. Elle est une théorie explicative mystifiante, mais l'auteur ne lui cherchera pas de racines dans le sous-sol des inférences cognitives, comme il s'y applique pour la religion. Car elle n'a pas d'existence possible dans une approche construite sur la dynamique positive de l'évolutionnisme, lequel a déjà beaucoup de mal à accepter dans son schéma tout ce qui n'est pas compétition pure et dure. Cependant, on peut noter que la psychologie et l'archéologie s'étant ralliées à la théorie de la sélection évolutionniste en ce qui concerne la dynamique des inférences cognitives, pensées, représentations, opinions et suppositions sur la conduite du partenaire, le vocabulaire s'enrichit de concepts récents comme la «sélection de parentèle» (p. 180) ou l'«altruisme réciproque» afin expliquer les "anomalies» observées par les éthologues: "Tout en étant individuellement désintéressé, l'altruisme réciproque reste égoïste du point de vue génétique. Les gènes responsables de ces stratégies ont une bonne chance de se propager même lorsque d'autres stratégies apparaissent dans le pool génétique» (p. 181).

Il y a là un tour de passe-passe : le modèle évolutionniste constate que les sociétés animales ont évolué par la compétition qui élimine les plus faibles et les moins aptes ; les humains, espèce particulière "de coopérateurs ", évoluent par compétition positive (mais pratiquent la sorcellerie). Lalliance inédite entre disciplines - l'axe de cette étude - maintient une fracture qui individualise l'être humain dans la continuité zoologique malgré l'ambition affichée de ne faire jouer que le niveau des réflexes excités par la compétition évolutionniste et qui seraient le seul ressort de la "sélection culturelle ». On retrouve ici les hypothèses de l'anthropologie cognitive élaborée par Dan Sperber, pour qui les mécanismes d'acquisition des connaissances et leur utilisation obéissent à une dynamique de type épidémiologique toute empreinte d'évolutionnisme automatique : seuls demeurent les réflexes et les inférences cognitives qui sont utiles, "pertinentes ", dans une situation donnée et facilement mises en œuvre, puis répétées et mémorisées, accédant au fil du temps à une forme biologique de "concepts stables". Pascal Boyer fait allusion à cette dynamique de la "pertinence " et mentionne le travail commun de Dan Sperber et de la linguiste Deirdre Wilson (p. 161) ${ }^{3}$. Après avoir cité la per-

2. Jeanne Favret-Saada, Les Mots, la mort, les sorts. La sorcellerie dans le Bocage, Paris, Gallimard, 1977 («Bibliothèque des Sciences humaines »).

3. Cf. Dan Sperber \& Deirdre Wilson, La Pertinence, communication et cognition (Paris, Éditions de Minuit, 1989 ; paru en anglais sous le titre Communication and Cognition, London, Blackwell, 1995), .../... 
tinence comme une de ses trois «boites à outils» (pp. 161-167), il en dévide longuement, dans sa conclusion, le fil conducteur pour construire une histoire de l'enrichissement des réflexes cognitifs appliqués à l'élaboration (la création) des agents religieux, dieux, esprits et ancêtres (pp. 308-326).

Un ressort essentiel de cette recherche sur les réflexes sociaux abondamment utilisé dans ce travail de synthèse est la qualité inconsciente de ces réflexes. Une tautologie qu'il semble nécessaire de rappeler tout au long du livre pour mieux combattre les illusions des travaux ethnologiques. Le passage du réflexe biologique à la construction cognitive puis à l'action sociale positive est situé, avec raison, dans le domaine précis que les ethnologues s'interdisent d'explorer en invoquant l'objectivité et la froideur scientifique. La biologie réglerait l'existence des inférences cognitives - via la puissance des émotions que l'individu éprouve comme un besoin d'action qui s'impose de façon irrésistible - au niveau infra-cognitif, ou plutôt, puisqu'on traite d'une espèce coopérante, pré-cognitif. S'agissant de contruire l'objet religion, l'ouvrage accorde son attention aux actions empreintes de moralité, une moralité recalibrée en altruisme réciproque et universel, puisque même les criminels (ceux des prisons américaines) reconnaissent l'immoralité de leurs actions. Et ainsi, une fois encore, l'auteur écarte de son champ de recherche les capacités de coopération négatives. La sorcellerie a déjà été qualifiée de «fantastique» (p. 191) et jugée définitivement inutile à la réflexion, car ambiguë (p. 267). En outre, de savantes études ont montré que les enfants, dès leur plus jeune âge, manifestent leur aptitude à un "réalisme moral où le "mal" paraît aussi intrinsèque au vol que la "rougeur" au coquelicot" (p. 178). Les aptitudes enfantines, même observées bien après l'apparition de la parole et quelques années de socialisation (pp. 174-178), viennent conforter la description du «sous-sol » de «l'espèce de coopérateur ».

Certes, conclut malicieusement Pascal Boyer, «cela peut sembler frustrant parce que la religion telle que je la définis ici ne serait qu'un effet secondaire du fonctionnement de notre cerveau, ce qui manque apparemment de grandeur » (p.325). En effet, le lecteur est dûment averti tout au long de la démonstration qu'il s'agit de réactions biologiques mobilisées par «la propension humaine à entretenir des pensées religieuses" (p. 326). Cette propension très multifonctionnelle à produire des « inférences » est bien le propre des coopérateurs dont la niche écologique est constituée par la circulation d'informations (p. 322). La qualité inconsciente de cette effervescence automatique est démontrée par le processus de sélection suivant: nous produisons en permanence des milliards d'informations, ou plutôt d'inférences, de suppositions, d'actes possibles, de solutions éventuelles et même de scénarios imaginaires plus construits, les "actions découplées " du réel, comme les jeux enfantins (p. 149) qui développent la fonction cognitive (p. 202). Dans cette activité fourmillante de notre cerveau-esprit « il y a beaucoup d'appelés et peu d'élus» (p. 325). Seules certaines inférences, parce qu'elles sont

ouvrage absent de la bibliographie. Dan Sperber est chargé par le CNRS, comme Pascal Boyer, de développer l'anthropologie cognitive en France. 
plus pertinentes, accèdent à l'état de concepts stables et seront éventuellement transmises par l'acquisition culturelle. Là s'arrête l'investigation de Pascal Boyer, au seuil de l'activité réfléchie et des normes sociopolitiques, abandonnées un peu dédaigneusement à l'anthropologue-ethnologue voué désormais à n'observer que les formes superficielles et mystifiantes, «secondaires ", de la variété culturelle.

L'épidémiologie des idées religieuses (pour parodier le titre d'un autre ouvrage de Dan Sperber brièvement évoqué) est ancrée dans des processus physico-chimiques comparables à un "ensemble de "mains invisibles" »: "La main invisible de la sélection culturelle fait en sorte que les concepts religieux acquis et transmis par les gens sont généralement plus aptes à les convaincre, étant donné ce qu’est leur existence " (ibid.). De la même façon, pourrait s'aviser un ethnologue de la Bourse, la main invisible du marché rend pertinents des choix d'achat et de vente parmi les milliards de suppositions faites sur les informations que posséderaient les autres actionnaires. Et tous ces réflexes cognitifs se coordonnent admirablement pour réguler le flux des capitaux et l'augmentation des dividendes dans un univers spéculatif en expansion que rien ne vient borner... jusqu'à la survenue d'un krach évidemment inopiné et expliqué ensuite par un dysfonctionnement de la régulation cognitive. La comparaison me semble justifiée à plus d'un titre, mais surtout parce que l'utilitarisme évolutionniste ne peut prospérer que dans un univers où il ne rencontre ni affrontement, ni ennemi, ni contradiction ou antagonisme. La main invisible est naturellement bienveillante et morale; ce qu'elle favorise est bon, bien et durable «étant donné ce qu'est » l'existence des gens (ibid.).

Comment une pareille main peut-elle laisser perdurer la sorcellerie? Nous avons eu la réponse en forme d'échappatoire. Une autre question est soulevée par l'auteur : comment tel rite de passage étudié au Cameroun peut-il favoriser la coopération sociale alors que les épreuves infligées aux jeunes gens, volontairement paradoxales, contribuent à créer «une sorte de flou cognitif»? (p. 242). C'est que «l'objectif officiel est plus complexe qu'il n'y paraît» (p. 243). Car, de toutes façons, les jeunes deviendront des adultes et la puissance de la dynamique de "coalition" organisera sans faille les conduites de coopération dans la chasse, la guerre et les mariages (ibid.). Qu'est devenue la complexité ? Comment la nécessité de pertinence, qui favorise la concaténation de réflexes la plus élémentaire, a-t-elle pu susciter des apprentissages basés sur la contradiction qui acquièrent, brièvement, un statut général puisque Pascal Boyer les place «au centre des initiations masculines» (p. 242) ? Pourquoi, en fin de compte, mentionner ce rituel paradoxal pour ne lui trouver aucune place dans la spéculation utilitariste, sinon admettre qu'il ne nuit pas à la dynamique de la "coalition " ${ }^{4}$ ?

Bien que les travaux ethnologiques soient assidûment renvoyés à leur faiblesse intrinsèque qui ne les différencie guère des aveuglements de la sociologie naïve, Pascal Boyer y recourt quand les informations dont il dispose lui semblent étayer

4. Coalition: tendance spontanée de l'espèce coopérative à s'organiser en groupes de concertation et de compétition (p. 120). Le modèle implicite des équipes de base-ball sous-tend les expériences américaines de psychologie sociale. La "coalition" est directement issue de l'activité cognitive taxonomique qui organise l'environnement en "catégories ontologiques» (p. 64). 
ses analyses. Il revient ainsi à plusieurs reprises sur l'existence des castes de forgerons de l'Afrique occidentale, puis de l'Afrique en général, bien qu'il remarque (p. 212) que ceux de l'Afrique centrale ne sont pas considérés comme des sources de pollution, la pollution étant le signe indiscutable d'une "essence" sociale (pp.133-134). I 1 suffit que certaines études (non référencées) aient parlé de castes d'artisans pour que l'observateur des processus dynamiques inconscients affirme que « dans la vie réelle $[. .$.$] les gens font partie de catégories sociales qui sont pré-$ sentées en termes essentialistes - castes de forgerons ou lignages - et ils les gèrent comme des coalitions » (p. 284). Non seulement la sociologie indiscutablement «naïve » est dans ce cas prise au sérieux, mais elle sert à démontrer la puissance de la transcendance qui surgit de ce découpage en groupes ressentis émotionnellement et cognitivement comme des entités naturelles (p. 282). L'exemple, qui se veut si démonstratif, des castes de forgerons est particulièrement mal venu car il fait l'impasse sur des siècles d'histoire et ignore totalement l'impact des premiers royaumes musulmans sur la situation politique de ces techniciens qui étaient autrefois des personnages considérables et considérés. Et qui le sont restés dans nombre de sociétés d'Afrique occidentale vivant sur les marges de ces royaumes (Minyanka, Kabiyè, Bassar, Tammariba...). Quant aux forgerons d'Afrique centrale, non seulement ils ne sont pas castés, mais ils étaient organisés en des sortes de compagnonages non lignagers, mobiles et politiquement actifs 5 .

Et c'est ainsi que, partant de la capacité taxonomique propre à l'être humain, qui ouvre brillamment ce livre, Pascal Boyer, via la puissance cognitive de la «coalition" (les bleus et les rouges des groupes expérimentaux de la psychologie sociale) arrive à nous faire admettre la transcendance de la courgette : "Nous supposons qu'il existe dans tout organisme en cours de développement quelque chose qui le pousse à devenir semblable aux autres membres de son espèce. Cette "essence" spécifique est ce qui permet aux courgettes et aux endives d'acquérir leur forme et leur goût spécifique» (p. 255). Les rituels, qui consistent en une suite d'actions (et qui seraient ainsi comparables à la croissance d'un plant de courgette), produisent un résultat inexplicable mais expliqué de la même façon que la transformation de la fleur en fruit, par un automatisme biologique et génétique. La transcendance du rituel ne differe pas de celle de la courgette car elle active les mêmes systèmes d'inférence cognitive. Cela ne surprendra pas le lecteur, sommé d'éviter les simplismes de l'ethnologie, qui a déjà rencontré l'essence des espèces animales identifiées par le chasseur-cueilleur, puis celle des "coalitions", des lignages et des castes. La transcendance est une application du système de causalité, un effet secondaire du fonctionnement de notre cerveau. Elle est donc présente partout où l'être cognitif exerce (ou subit ?) l'activité de ses systèmes d'inférence. L'ethnologue, incurablement naïf mais obstiné, estime qu'une question importante est ainsi abordée : pourquoi l'espèce des coopérants est-elle amenée à recon-

5. Cf. Colleen Kriger, Pride of Men. Iron working in 19th Century West Central Africa, Portsmouth, N. H. Heineman - Oxford, James Currey - Cape Town, David Philip, 1999 ( Social History of Africa ). [Voir le compte rendu de cet ouvrage par Marie-Claude Dupré dans L'Homme, 2001, 160 : 261-264. Ndlr.] 
naitre de la transcendance dans la récurrence des manifestations observables dans son environnement, que ce soit une antilope, une courgette, un rituel ou, plus impalpable, un sorcier, un esprit d'ancêtre ou une divinité ? Ce serait là, comme on nous l'a déjà dit, le résultat de notre " propension » à entretenir des pensées religieuses (p. 326). L'homme cognitif voit des dieux partout.

Pour Pascal Boyer, la somme des avancées de la psychologie cognitive appliquée au développement de l'enfant et à l'évolution humaine est si novatrice et si excitante intellectuellement qu'il en oublie de la confronter aux avancées récentes, certes plus grossières mais nombreuses, de l'anthropologie ethnologique. Il s'enferme dès lors dans une réflexion qui restreint son attention aux seuls développements des autres branches de la recherche cognitive. Si bien que le corpus d'informations ethnologiques sur lesquelles il s'appuie pour dépasser la simplicité de leurs résultats et accéder à une construction rigoureuse est plus proche du postulat que de la réalité. En effet, puisqu'il définit l'approche ethnologique par son aveuglement, qu'importe si certains forgerons ne sont pas castés puisque beaucoup le sont (et certainement de toute éternité), qu'importe si la sorcellerie existe du moment qu'on peut la considérer comme un simple aspect de la coopération positive. La capacité des maîtres-initiateurs à mettre les jeunes en contact avec les émotions puissantes et contre-intuitives que sont les ordres contradictoires et les manipulations paradoxales est mentionnée par acquit de conscience, sans même renvoyer le lecteur français à la bibliographie francophone ${ }^{6}$. Pourtant ces ouvrages, tout comme la réglementation tatillonne et rigoureuse de la chasse au filet, démontrent chez les sociétés "naïves " un grand savoir-faire dans le recours à des processus infra-conscients que l'anthropologie cognitive se vante d'avoir tout juste découverts... Mais il suffit à l'auteur d'énumérer quelques exemples choisis qui doivent leur aspect de généralité à leur ancienneté, comme si la patine était une garantie de vérité, qu'il s'agisse de spéculations sur les hommes préhistoriques ou de travaux ethnologiques publiés il y a vingt ans et davantage. Ce recours sélectif, généralisant et non actualisé, aux travaux des ethnologues fait que l'ensemble de la construction, si ambitieuse soit-elle, n'est pas « réfutable».

Les termes (qui sont des concepts) d'ambivalence, d'ambiguïté, de contradiction et de paradoxe sont mentionnés sans être examinés dans leur dynamique cognitive, car ils rendraient évidente la pratique efficace (et non pas simplement naïve) des mécanismes sociaux de la construction et de la transmission de connaissances en tous genres. Pire, ils infirmeraient totalement la dynamique d'épidémiologie et de pertinence utilitariste qui fait l'impasse sur les contrôles sociaux du «sous-sol» neuronal. La théorie de la pertinence est calquée sur la spéculation mystificatrice des opérations boursières dans le contexte particulier de la mondialisation libérale, construction fictive s'il en est.

6. C'est le seul exemple qui admet une dynamique complexe des automatismes cognitifs. Toutefois, la bibliographie omet de mentionner deux articles de Michael Houseman qui seraient accessibles au lecteur francophone. Cf. Michael Houseman, "La double contrainte et le paradoxe rituel ", Cahiers critiques de thérapie familiale et des pratiques de réseaux, 1992, $14: 155-163$, et "Les artifices de la logique initiatique ", Journal des africanistes, 1984, 54 (1) : 41-66. 
En lisant ce livre, on se demande si l'auteur, directeur de recherche au CNRS, cherche bien à développer l'anthropologie cognitive en France. Il semble qu'il s'efforce plutôt d'importer des concepts et des exemples à peine transposés, où Ms Smith devient Mme Durand (pp. 24-25). Sans s'apercevoir que la partie réflexive de la "pertinence ", inséparable du contexte social, intervient très tôt dans l'automatisme des réflexes cognitifs. Voulant à tout prix cantonner l'activité réflexe inconsciente dans ses automatismes dénués de réflexion, Pascal Boyer semble succomber à l'utilitarisme moral anglo-saxon qui imprègne (inconsciemment ?) les protocoles d'expérience en psychologie sociale.

MOTS CLÉS/KEYWORDS: anthropologie cognitive/cognitive anthropology - systèmes d'inférence/inferential systems - utilitarisme moral/utilitarianism - propension religieuse/religious propensity. 